\title{
Percepción social de los problemas ambientales en Yucatán, México \\ UNA VISIÓN DESDE LA GEOGRAFÍA
}

DOI: $10.22403 /$ UROOMX/TYP09/02

Francisco Bautista

Centro de Investigaciones en Geografia Ambiental,

Universidad Nacional Autónoma de MÉxico

Amira M. Balancán-Zapata

Jorge Navarro-Alberto

Universidad Autónoma de Yucatán

Gerardo Bocco

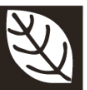

Centro de Investigaciones en Geografia Ambiental

Universidad Nacional Autónoma de MÉxico

\section{RESUMEN}

A partir de la filosofía de "pensar de manera global, pero actuar de manera local", y de que la primera acción debería ser la elaboración de diagnósticos ambientales sociales, el objetivo de este trabajo fue medir la percepción de la sociedad en torno a los problemas ambientales del estado de Yucatán. Se aplicaron 195 encuestas y se elaboró una base de datos con ocho variables. Los análisis de datos se llevaron a cabo a través del cruce de variables, estadísticos de asociación y ji cuadrado. Los tres principales problemas ambientales percibidos por la sociedad fueron: contaminación del acuífero, erosión de playas y pérdida de biodiversidad.

La población encuestada, e incluso los expertos, es de procedencia urbana e identifica plenamente los problemas ambientales de la ciudad de Mérida y de algunos municipios costeros aledaños. Otras zonas tienen prioridades diferentes en cuanto a sus problemas ambientales. El enfoque geográfico transdisciplinario adoptado permitió valorar en su justa dimensión los resultados de este estudio, además de que se hizo notar que, en la percepción de los problemas ambientales, los individuos tienen un sesgo geográfico por el conocimiento de su entorno.

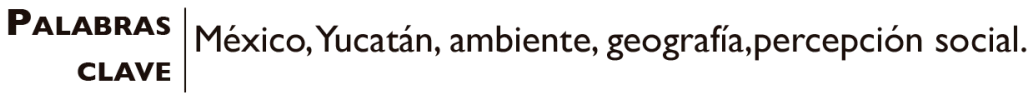

Correos electrónicos: leptosol@ciga.unam.mx•abalzapa@uady.mx•jorge.navarro@uady.mx• gbocco@ciga.unam.mx 


\section{Introducción}

Los problemas ambientales del mundo contemporáneo son esencialmente problemas sociales. En los últimos años, en el plano mundial, tanto la ocupación y modificación del territorio como la obtención intensiva de recursos de los ecosistemas y su consecuente transformación y, en algunos casos, degradación, se ha dado de manera tal que no tiene precedente en la historia de la humanidad.' En esta modalidad del desarrollo, en realidad a veces un mero crecimiento del producto bruto, se produce una sobreexplotación del capital natural, con la finalidad de obtener la máxima ganancia y el excedente económico en el corto plazo. Este modelo es el causante de fenómenos ambientales globales (laemisión de gases de efecto invernadero, la destrucción de la capa de ozono, la contaminación atmosférica y de los océanos, entre otros). En buena medida, es también el causante de problemas que se operan y se perciben en el ámbito local: deforestación, erosión de suelos, pérdida de la biodiversidad, disminución del suministro y deterioro de la calidad del agua potable, etc. (Ramírez, 1997). A su vez, todo esto se convierte en un límite para el modelo de desarrollo y amenaza la posibilidad de ensayar modelos alternos (Bautista et al., 2000).

La intensidad de la ocurrencia de los problemas ambientales y la cauda de consecuencias sociales y económicas son inversamente proporcionales al nivel de bienestar económico de las clases sociales; así, las poblaciones más pobres son, por lo general, las más vulnerables y afectadas. Los desastres tales como las inundaciones y deslaves se presentan en las zonas donde se localizan las viviendas y la infraestructura de la población más pobre. Otros procesos, en cambio, afectan de manera equitativa a todos los sectores sociales, por ejemplo el calentamiento global y sus repercusiones: el incremento del nivel de los mares, los cambios en los ecosistemas y en los sistemas agrícolas.

Es conveniente disponer de un conocimiento lo más acabado posible de los factores sociales que provocan -y han provocado en el pasado- la conversión, degradación y, en ocasiones, destrucción de los ecosistemas. Si aceptamos como válida la premisa de "pensar de manera global, pero actuar de manera local", es preciso admitir que se requiere dedicar esfuerzos científicos a ambos niveles. La geografía, por su particular y tradicional visión de los problemas ambientales, ofrece una perspectiva de trabajo robusta para estos fines. Desde el punto de vista de ciencia aplicada a la búsqueda de soluciones $y$, en forma

'Véase, por ejemplo, Leff y Carabias, 1993.

TeOría y Praxis

9 (201 I: 33-54) 
complementaria, del enfoque de ciencia comprometida socialmente, resulta viable dedicar esfuerzos de investigación, en especial participante, al nivel local. Por ejemplo, Eden (1998) sostiene que la investigación científica en geografía debe tener como meta no sólo la producción de conocimiento ambiental, sino el compromiso con la negociación de la política ambiental.

Es necesario establecer estrategias claras orientadas al manejo de la incertidumbre, siempre presente en temas tan complejos como lo ambiental, para de este modo sistematizar la información disponible y hacerla accesible a los tomadores de decisiones (de organizaciones sociales y gubernamentales). Sin embargo, es importante desmitificar el poder omnímodo de las bases de datos digitales y evaluarlas de forma crítica ante las pretensiones de objetividad de los datos estadísticamente elaborados y derivados, por ejemplo, de cartografía digital, controlando así intentos de manipulación de los datos y la información que de ellos resulta.

Una estrategia fundamental es la elaboración de diagnósticos ambientales locales. A menudo la estrategia es unidireccional: a) diagnóstico ambiental realizado por expertos; $b$ ) toma de decisiones en el ámbito gubernamental (municipal, estatal o federal); c) desarrollo del proyecto; d) comunicación de resultados en el nivel social;y e) puesta en marcha del proyecto (Espinoza, 2002; Semarnat, 1996b). Esta forma de trabajo, que no involucra desde el diagnóstico a la sociedad, tiene altas probabilidades de no alcanzar su objetivo, ya que, con su comportamiento, actitud y desempeño, es la gente quien finalmente decide el éxito o fracaso de los programas ambientales.

El propósito de este ensayo es medir, a manera de un primer diagnóstico ambiental social, la percepción social en torno a los principales problemas ambientales del estado de Yucatán. La premisa es que toda problemática ambiental está espacialmente distribuida, de tal suerte que un enfoque geográfico puede contribuir tanto a formular con mayor precisión las preguntas clave, como a ofrecer respuestas que consideren aspectos del ámbito biofísico y del social de forma integrada. Ésta es una de las fortalezas del enfoque geográfico en cuestiones ambientales.

Si bien la geografía ambiental es un campo aún incierto desde el punto de vista epistémico, la contribución de la geografía a la investigación teórica y práctica en temas ambientales ha sido importante a lo largo de las últimas décadas (Bocco, 2007). Como ya se apuntó, las nuevas tendencias en 
investigación ambiental sugieren ensayar enfoques participantes derivados de algún grado de compromiso social por parte de los académicos. La participación de la sociedad en la elaboración de los diagnósticos ambientales genera responsabilidad política; permite racionalizar las controversias y resolver disputas; da transparencia a la toma de decisiones y logra mayor aceptación de éstas; disminuye los errores y los actos de corrupción; y fortalece las instituciones. Todas estas ventajas de los diagnósticos ambientales sociales deberían ser aprovechadas por los tres niveles de gobierno para realizar una toma de decisiones asertiva.

\section{Materiales y métodos}

Se empleó una fuente de investigación primaria directa, la encuesta estructurada; se aplicaron 195 cuestionarios, en la ciudad de Mérida, en el estado de Yucatán (figura I), durante febrero y marzo de 2006. Se utilizó un muestreo al azar. Los entrevistadores fueron estudiantes de la licenciatura en Biología, que fueron capacitados mediante un taller. En las encuestas se solicitó información sobre: a) la población encuestada;b) la identificación de los problemas ambientales;c) el compromiso de la sociedad con la solución de los problemas identificados; d) la identificación de los responsables de atender los problemas ambientales; y e) la reasignación del gasto público para atender los problemas ambientales (cuadro I).

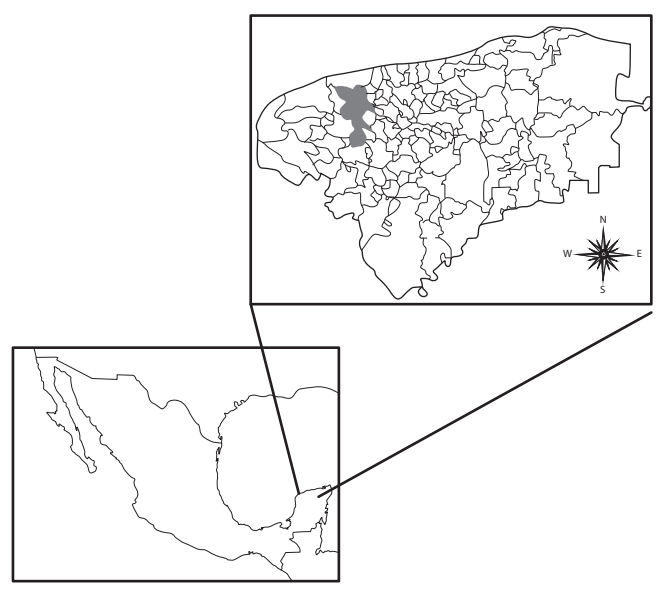

Figura I. Localización del Área de ESTUdio 


\section{Cuadro I. Preguntas de la encuesta ambiental}

I. Profesión / Ocupación.

2. Edad

3. ¿Cuáles son los tres principales problemas ambientales en Yucatán?

4. ¿Cuánto dinero esta dispuesto a aportar a un fondo para la solución de los problemas ambientales en Yucatán?

5. ¿Por cual problema ambiental esta dispuesto a manifestarse socialmente?

6. ¿A quién le corresponde remediarlos?

7. ¿A qué rubro le quitaría dinero para destinarlo a la solución de los problemas ambientales?

$\square$ Salud $\square$ Educación $\square$ Policía $\square$ Bomberos $\square$ Servicios urbanos $\square$ Otros

Las dos primeras preguntas se hicieron con el objetivo de caracterizar a la población encuestada. La tercera fue utilizada para identificar los problemas ambientales (al encuestado se le pidió enlistar los tres principales problemas ambientales de Yucatán). Las preguntas cuatro y cinco estuvieron dirigidas a identificar el nivel de compromiso del encuestado con la solución de los problemas ambientales. La pregunta seis se planteó con el fin de que el encuestado identificara al responsable de encontrar la solución a los problemas ambientales. Un argumento muy usado para justificar las escasas acciones en contra de los problemas ambientales es la falta de presupuesto, por lo cual, se realizó la pregunta siete, considerando que el encuestado fuera quien tomara la decisión.

Una vez aplicada la encuesta, se capturó y depuró la información en una hoja de cálculo. La base de datos estuvo conformada por los 195 entrevistados y ocho variables: ocupación, edad, principales problemas ambientales percibidos, disponibilidad de aportación económica, cantidad monetaria de aportación, responsabilidad para la solución de problemas ambientales, intención de manifestación pública y reasignación del gasto público. El análisis de datos se llevó a cabo mediante el cruce de variables, estadísticos de asociación y ji cuadrado, para medir el grado de la percepción de los individuos sobre los problemas ambientales y la disponibilidad para apoyar propuestas de solución, que podían explicarse en función de la ocupación y edad de los encuestados.

Las respuestas numéricas (e.g., monto de aportación al fondo) se analizaron a través de pruebas no paramétricas debido a que presentaron observaciones muy extremas. Para explorar con mayor detalle nos preguntamos si la 
distribución de respuestas positivas respecto a la disponibilidad de aportar dinero es independiente de la ocupación del entrevistado. Para ello se realizó una prueba ji cuadrado de independencia. En todos los análisis se empleó el programa SPSS 12.0 (SPSS 2003), con un nivel de $5 \%$ como máximo para declarar cualquier asociación o diferencia como significativa.

Los resultados de las encuestas se compararon con las opiniones de expertos, para lo cual en septiembre de 2006 se realizó un taller con los participantes del Programa Institucional del Medio Ambiente (PIMA) de la Universidad Autónoma de Yucatán. Los expertos son de las siguientes áreas: Medicina, Ingeniería, Ecología, Química y Educación. Las técnicas utilizadas en el taller fueron lluvia de ideas, agrupamiento de ideas y discusión de los problemas ambientales que hay en Yucatán para ordenarlos por prioridad (Santos y Molina, 2004).

Los resultados obtenidos mediante las encuestas y el taller de expertos se compararon con los problemas ambientales de Yucatán identificados por otros estudios que han sido publicados.

\section{Resultados}

Características de la población entrevistada

La muestra de estudio presentó las siguientes características demográficas: $42 \%$ fueron estudiantes de licenciatura, $24 \%$ profesionistas, $14 \%$ empleados, $9 \%$ amas de casa, $6 \%$ comerciantes y un $5 \%$ dedicado a otras actividades. La distribución de las edades fue: $57 \%$ de las personas se encontraba en un intervalo de 18 a 25 años, $12 \%$ de 36 a 45 años, I I \% de 26 a 35 años y el $10 \%$ restante con edades de 50 años en adelante.

Identificación de los problemas ambientales

Como se puede apreciar en el cuadro 2, los tres principales problemas ambientales percibidos por la sociedad son: la contaminación del acuífero, la erosión de playas y la pérdida de biodiversidad, con ponderaciones de $2 \mathrm{I} . \mathrm{I}$, I 8.8 y $\mathrm{I} 2.2 \%$, respectivamente. Dicho de otra forma, con 6I, 55 y 35 $\%$ de menciones por el público, con $95 \%$ de confianza. Pero aun siendo tres problemas los de mayor importancia, la lista incluye un total de 19 problemas ambientales identificados. 
Cuadro 2. Principales problemas ambientales identificados en Yucatán

\begin{tabular}{lrrcc}
\hline Problemas ambientales & $\begin{array}{c}\text { Total } \\
\text { casos }\end{array}$ & $\begin{array}{c}\text { Total } \\
(\%)\end{array}$ & $\begin{array}{c}\text { Por caso } \\
(\%)\end{array}$ & EE $^{\prime}$ \\
\hline Contaminación del acuífero & 120 & 21.13 & $6 \mathrm{I}$ & 0.035 \\
Erosión del playas & 107 & 18.84 & 55 & 0.036 \\
Pérdida de la biodiversidad & 69 & 12.15 & 35 & 0.034 \\
Degradación de la capa de ozono & 56 & 9.86 & 29 & 0.032 \\
Agotamiento del agua & 47 & 8.27 & 24 & 0.030 \\
Cambio climático & 44 & 7.75 & 22 & 0.030 \\
Erosión del suelo & 33 & 5.81 & 17 & 0.027 \\
Basura & 33 & 5.81 & 17 & 0.027 \\
Pérdida de fertilidad en suelos & 28 & 4.93 & 14 & 0.025 \\
Desertificación & 11 & 1.94 & 6 & 0.016 \\
Deforestación & 5 & 0.88 & 3 & 0.011 \\
Aire & 4 & 0.70 & 2 & 0.010 \\
Ruido & 3 & 0.53 & 2 & 0.009 \\
Pobreza & 2 & 0.35 & 1 & 0.007 \\
Pozos petroleros & 2 & 0.35 & $\mathrm{I}$ & 0.007 \\
Incendios forestales & $\mathrm{I}$ & 0.18 & $\mathrm{I}$ & 0.005 \\
Mares & $\mathrm{I}$ & 0.18 & $\mathrm{I}$ & 0.005 \\
Contaminación de playas & $\mathrm{I}$ & 0.18 & $\mathrm{I}$ & 0.005 \\
Quemas & $\mathrm{I}$ & 0.18 & $\mathrm{I}$ & 0.005 \\
Total & 568 & 100 & & \\
\hline
\end{tabular}

'EE= Error estándar.

Con respecto a la contaminación del acuífero hubo valiosas observaciones, como la abundancia de este recurso, pero el riesgo que corre por la falta de drenaje (García y Álvarez, 2003) y también por la facilidad con la que se contamina, por las características del ambiente, al contar con mantos freáticos muy superficiales. Ihl et al. (2007) reportan planicies menores de $40 \mathrm{msnm}$ en $80 \%$ del territorio de Yucatán; mientras que Bautista et al. (2005) señalan que los suelos son someros (Leptosol) en $80 \%$ del estado, de lo que se deduce una escasa protección del acuífero, que es una de las múltiples funciones del suelo (Bautista, 2005). 
Compromiso del entrevistado con la solución de los problemas ambientales

De los entrevistados, 78.5 \% está dispuesto a aportar recursos económicos en favor de acciones que prevengan o remedien los problemas ambientales; sin embargo, la mayoría aclaró que únicamente se comprometería si el gobierno fuera el responsable directo de organizar las acciones tendientes a resolver los problemas. En otras palabras, las aportaciones económicas se harían sólo a un fondo o programa gubernamental.

El coeficiente de incertidumbre (medida de asociación entre las dos variables nominales $=$ aportación y ocupación), asumiendo que la respuesta de aportación es la variable dependiente, fue igual a 0.020 , con un valor $P=0.036$, lo cual indica que la disponibilidad de aportación depende de la ocupación. Explorando los residuos estandarizados, se observa que las categorías que no concuerdan con la distribución total de las respuestas ( $21.5 \%=\mathrm{No}, 78 \%=\mathrm{Si})$ fueron las amas de casa y los comerciantes, quienes dieron respuestas que se pueden catalogar como no distintas (gráfica I).

Las cantidades reportadas por los encuestados presentan mucha variabilidad, desde la "no disponibilidad" de aportar monto alguno en favor de la solución de los problemas ambientales, hasta cantidades cercanas o iguales a los 1000 pesos. Estas cantidades altas son valores extremos en comparación con las aportaciones restantes. No obstante, cuando se analizan los montos medianos, éstos no difieren significativamente respecto de la ocupación (ji cuadrado de la prueba de Kruskal-Wallis $=7.903, \mathrm{gl}=4, \mathrm{P}=0.095)($ figura $2 \mathrm{~A})$, ni tampoco respecto de las edades de los encuestados (ji cuadrado de KruskalWallis $=8.249, \mathrm{gl}=5, \mathrm{P}=0 . \mathrm{I} 43$ (figura $2 \mathrm{~B}$ ). Aquí se eliminó una categoría de edad en el análisis (60-69), porque solamente hubo un individuo en ella. La aportación mediana global es de 50 pesos.

\section{Manifestación pública}

De la población encuestada, $82.1 \%$ está dispuesta a manifestarse públicamente para lograr la solución de los problemas ambientales. La contaminación del acuífero, la basura y la erosión de las playas fueron los tres principales asuntos ambientales que motivarían las protestas públicas. Cabe resaltar que el manejo de la basura alcanzó el lugar número ocho en importancia como prioridad, pero fue el número dos que motivaría la protesta ciudadana, lo cual ocurrió porque, para las amas de casa, el asunto del manejo de la basura es prioritario. Por su parte, $19.9 \%$ de los encuestados no participaría en esta clase de protestas ciudadanas. 
Bautista, Balancán-Zapata

Navarro-Alberto, Bocco

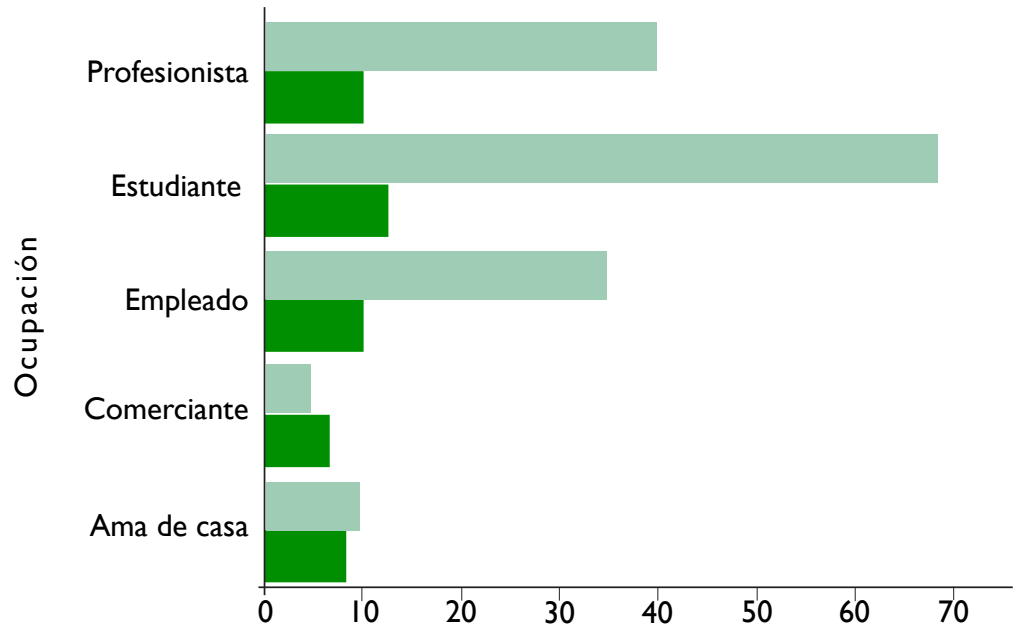

GRÁFICA I. DISPONIBILIDAD DE APORTACIÓN ECONÓMICA PARA SOLUCIONAR PROBLEMAS AMBIENTALES SEGÚN LA OCUPACIÓN

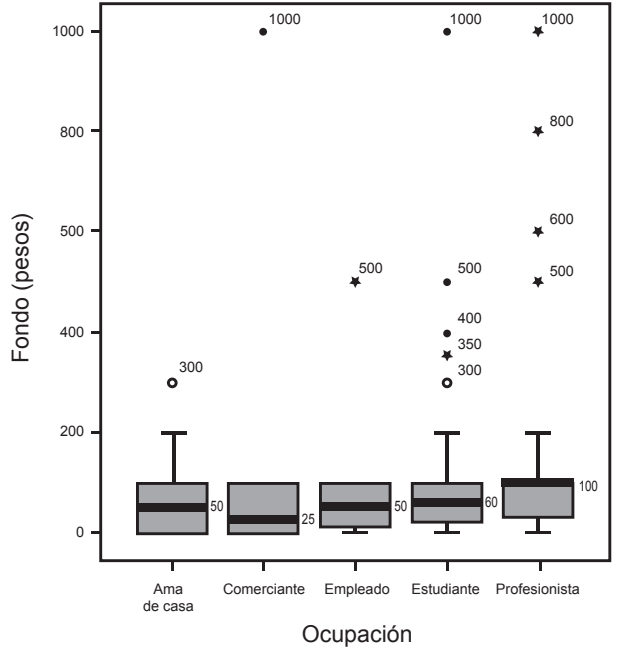

A

Figura 2. Diagramas de CAJA de aPORTACIONES AL FONDO PARA LA SOLUCIÓN DE LOS PROBLEMAS AMBIENTALES. $A=$ OCUPACIÓN, $B=$ EDAD. LAS LÍNEAS HORIZONTALES DENTRO DE CADA CAJA INDICAN LAS MEDIANAS; LOS CÍRCULOS, VALORES EXTREMOS MODERADOS; Y LOS ASTERISCOS, VALORES EXTREMOS.

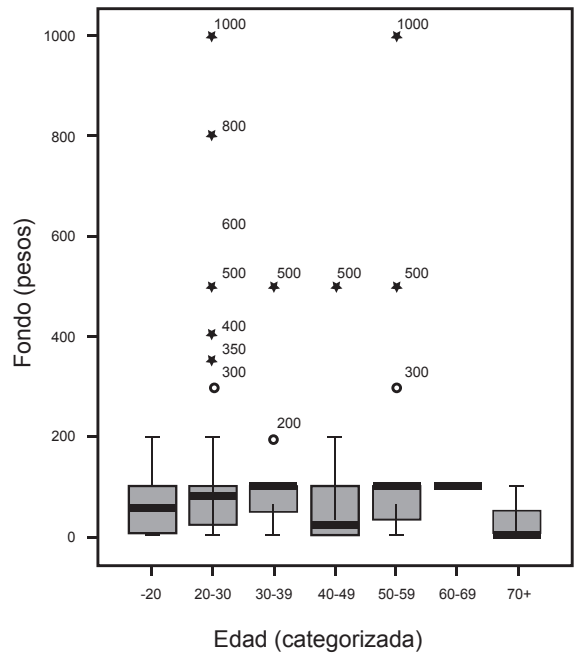

B 
Percepción social de los problemas ambientales en Yucatán

En cuanto a la asociación entre la edad del encuestado y su disponibilidad para manifestarse públicamente en pro de la solución de los problemas ambientales, en todos los grupos de edad se observó la tendencia a presentar una mayor proporción de encuestados que se manifestarían públicamente que de aquellos que no lo harían (cuadro 3). El valor de ji cuadrado no es significativo (ji cuadrado $=10.164, \mathrm{P}$ de la prueba exacta $=0.122$ ); más aún, la medida de incertidumbre, igual a 0.057 (suponiendo a la manifestación dependiente de la edad), no es significativamente distinta de $0(P=0.119)$. Por tanto, hay evidencias de que la decisión de manifestarse es independiente de la edad (quizás salvo en el grupo de edad de $70+$, donde dos de tres individuos

Cuadro 3. Categorías de edAd y su disposición a manifestarse PÚBLICAMENTE PARA LA SOLUCIÓN DE LOS PROBLEMAS AMBIENTALES

\begin{tabular}{|c|c|c|c|c|}
\hline \multirow[b]{2}{*}{ Edad } & & \multicolumn{3}{|c|}{ Intención de manifestación pública } \\
\hline & & No & $\mathrm{Si}$ & Total \\
\hline \multirow{2}{*}{$<20$} & Número & I & 27 & 28 \\
\hline & (\%) & 3.6 & 96.4 & 100 \\
\hline \multirow{2}{*}{$20-29$} & Número & 19 & 76 & 95 \\
\hline & (\%) & 20 & 80 & 100 \\
\hline \multirow{2}{*}{$30-39$} & Número & 4 & 11 & 15 \\
\hline & (\%) & 26.7 & 73.3 & 100 \\
\hline \multirow{2}{*}{$40-49$} & Número & 5 & 27 & 32 \\
\hline & $(\%)$ & 15.6 & 84.4 & 100 \\
\hline \multirow{2}{*}{$50-59$} & Número & 4 & 17 & 21 \\
\hline & (\%) & 19 & 81 & 100 \\
\hline \multirow{2}{*}{$60-69$} & Número & 0 & 1 & I \\
\hline & $(\%)$ & 0 & 100 & 100 \\
\hline \multirow[t]{2}{*}{$70+$} & Número & 2 & I & 3 \\
\hline & (\%) & 66.7 & 33.3 & 100 \\
\hline \multirow[t]{2}{*}{ Total } & Número & 35 & 160 & 195 \\
\hline & (\%) & 17.9 & 82.1 & 100 \\
\hline
\end{tabular}


indicaron no manifestarse, pero estos casos son tan pocos que casi no afectan el análisis de asociación y el estadístico ji cuadrado).

No se observaron diferencias significativas entre ocupaciones respecto a la proporción de personas que se manifestarían públicamente y las que no (ji cuadrado $=8, P=0.088$ ) (cuadro 4 ). La asociación entre estas dos variables no es significativa (coeficiente de incertidumbre $=0.039, P=0.159$ ). De los entrevistados, $\mathbf{8 2 . 1} \%$ admite manifestarse públicamente, una proporción que comparten en forma aproximada los empleados, estudiantes y profesionistas. Aunque en los comerciantes la relación es alrededor de I:I, y la mayoría de las amas de casa (94.I \%) aceptaría manifestarse, estas discrepancias no son lo suficientemente grandes como para dominar la tendencia similar de las otras tres ocupaciones.

CuAdro 4. OCupaciones de los ENCUESTAdOS Y SU DISPOSICIÓN A MANIFESTARSE PÚBLICAMENTE PARA LA SOLUCIÓN DE LOS PROBLEMAS AMBIENTALES

\begin{tabular}{|c|c|c|c|c|c|}
\hline & & & Manifes & & Total \\
\hline & & & No & $\mathrm{Si}$ & \\
\hline Ocupación & Ama de casa & Número & 1 & 16 & 17 \\
\hline & & (\%) & 5.9 & 94.1 & 100 \\
\hline & Comerciante & Número & 5 & 6 & II \\
\hline & & (\%) & 45.5 & 54.5 & 100 \\
\hline & Empleado & Número & 9 & 34 & 43 \\
\hline & & (\%) & 20.9 & 79.1 & 100 \\
\hline & Estudiante & Número & 12 & 67 & 79 \\
\hline & & (\%) & 15.2 & 84.8 & 100 \\
\hline & Profesionista & Número & 8 & 37 & 45 \\
\hline & & (\%) & 17.8 & 82.2 & 100 \\
\hline & Total & Número & 35 & 160 & 195 \\
\hline & & (\%) & 17.9 & 82.1 & 100 \\
\hline
\end{tabular}

Responsables de lograr la solución de los problemas ambientales

De los entrevistados, 53 \% responsabiliza a la industria de los problemas de contaminación; $30 \%$, al gobierno, ya que de éste depende que sean respetados 
los lineamientos, normas y políticas ambientales;y $17 \%$ a la sociedad en general, la cual también debe contribuir al buen uso de los recursos naturales con los que cuenta (cuadro 5). Los encuestados comentaron que es necesario mejorar la educación ambiental en los hogares y en las escuelas, para que realmente exista una cultura y una conciencia de respeto y cuidado del ambiente.

El número de personas que señaló a la sociedad como responsable de lograr la solución de los problemas ambientales fue muy similar al número que respondió que las autoridades deberían ser las encargadas de ello; lo cual se confirma con la prueba ji cuadrado de bondad de ajuste de una razón I:I entre los dos grupos de responsables (ji cuadrado $=0.046, \mathrm{~g} I=\mathrm{I} P=0.886$ ).

Se encontró una asociación marginal entre la ocupación del encuestado y su opinión sobre el sector responsable de la solución de los problemas ambientales: ji cuadrado $=9.480, \mathrm{P}=0.049$; coeficiente de incertidumbre (responsables como variable dependiente) $=0.036, P=0.054$. Una mayor proporción de amas de casa, comerciantes y empleados señalaron a las

CuAdRO 5. TABLA DE CONTIGENGENCIA ENTRE OCUPACIÓN Y RESPONSABLES DE LOGRAR LA SOLUCIÓN DE LOS PROBLEMAS AMBIENTALES

\begin{tabular}{lcrrr}
\hline \multirow{2}{*}{ Ocupación } & & \multicolumn{2}{c}{ Responsables } & \\
& & Autoridades & Sociedad & Total \\
\hline Ama de casa & Número & 12 & 5 & 17 \\
Comerciante & $(\%)$ & 70.6 & 29.4 & 100 \\
& Número & 7 & 4 & 11 \\
Empleado & $(\%)$ & 63.6 & 36.4 & 100 \\
& Número & 26 & 17 & 43 \\
Estudiante & $(\%)$ & 60.5 & 39.5 & 100 \\
& Número & 33 & 46 & 79 \\
Profesionista & $(\%)$ & 41.8 & 58.2 & 100 \\
& Número & 18 & 27 & 45 \\
Total & $(\%)$ & 40 & 60 & 100 \\
& Número & 96 & 99 & 195 \\
& $(\%)$ & 49.2 & 50.8 & 100 \\
\hline
\end{tabular}


autoridades como las responsables de lograr la solución de los problemas ambientales, en tanto que los estudiantes y profesionistas se inclinaron más por la sociedad (cuadro 5). Estas diferencias parecen explicar la asociación marginalmente significativa entre ocupación y responsabilidad.

El discurso gubernamental de que la sociedad es la principal responsable no ha sido incorporado ni asumido por la población encuestada, lo cual implica que una parte de ésta es consciente de que existen verdaderos responsables directos de esta situación.

Reasignación del gasto público para solucionar los problemas ambientales

La pregunta se realizó pensando que, si bien todos queremos el mejoramiento del ambiente, la cantidad de dinero con la que se cuenta siempre será finita y se requerirá reasignar el gasto público. Por las respuestas de los entrevistados queda claro que la mayoría de la gente preferiría el mejoramiento del ambiente que los subsidios económicos a la iniciativa privada. La diferencia entre las frecuencias observadas y las esperadas bajo el supuesto de equiprobabilidad de respuestas en las categorías de reasignación es significativa (ji cuadrado $=$ 348. I7, P <0.00 I) (gráfica 2).

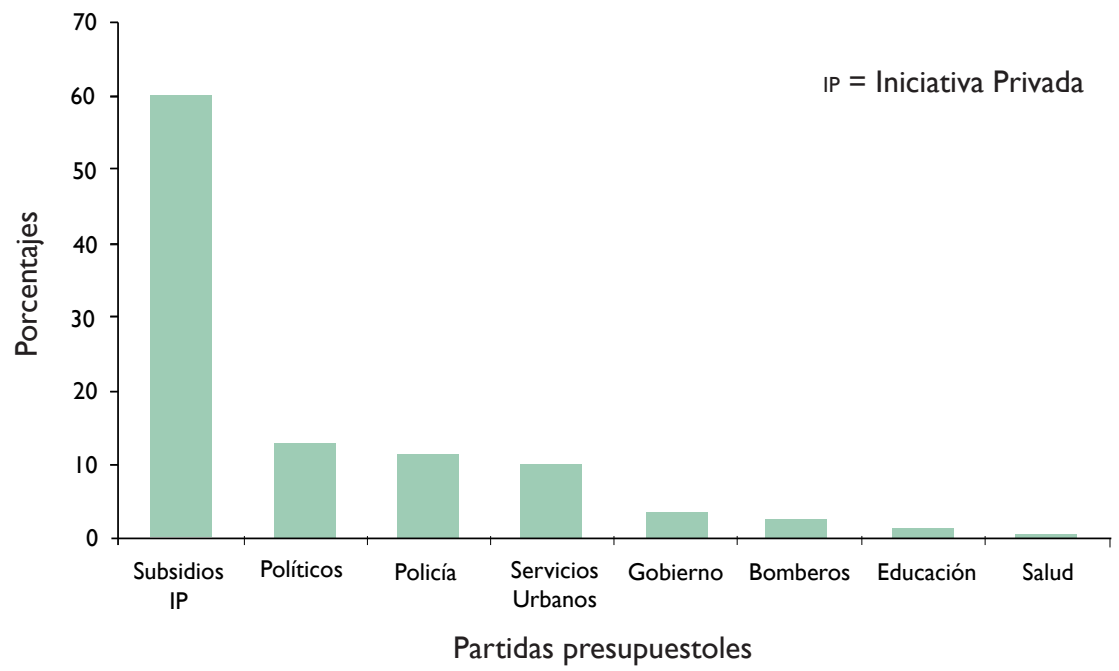

Gráfica 2. Selección de LAS PARTIDAS PRESUPUESTALES ELIMINADAS O REDUCIDAS PARA REASIGNAR EL GASTO PÚBLICO AL MEJORAMIENTO DEL AMBIENTE. 
Con relación a la reasignación de partidas presupuestales, $59.5 \%$ de los encuestados sugiere que los subsidios a la iniciativa privada se eliminen o recorten para dedicar recursos económicos a la solución de problemas ambientales. Esto contrasta con la baja frecuencia de selección de las otras partidas presupuestales.

Los apoyos a la iniciativa privada se otorgan, según el discurso gubernamental, para el mejoramiento del empleo y en general de las actividades productivas. Los resultados de este trabajo sugieren que la gente considera que este tipo de subsidios sólo es para algunos sectores de la sociedad, en cambio, el mejoramiento del ambiente es para la sociedad en su conjunto.

\section{Taller de expertos}

Los problemas identificados por el panel de expertos con la lluvia de ideas son: manejo de desechos sólidos no peligrosos; contaminación del manto freático; deforestación y pastizales; contaminación del suelo por agroquímicos; sobreexplotación de especies en veda; falta de planeación de la urbanización; contaminación atmosférica; emisiones de gases al medio ambiente; contaminación; modificación del hábitat; desastres naturales como consecuencia de problemas ambientales; alteración de la flora y fauna por prácticas inadecuadas; incorporación de sustancias tóxicas en la cadena alimentaria; uso irracional del agua; falta de planeación para el saneamiento ambiental; falta de infraestructura para el manejo de residuos peligrosos; contaminación por ruido; falta de educación y conciencia ambiental; ahorro de energía, agua y papel (reciclaje); contaminación costera; disminución de áreas selváticas; aumento de cobertura de agua potable; tratamiento de aguas residuales y claras; descargas de aguas y sólidos porcícolas; y uso de energía fósil.

Al agrupar los problemas ambientales y priorizarlos el resultado fue: I) contaminación del agua; 2) uso excesivo de recursos naturales, como modificación del hábitat y deforestación; 3) residuos sólidos y peligrosos; y 4) escasa educación ambiental. Es importante mencionar que las quemas no se vislumbran como un problema ambiental, aun cuando la publicidad gubernamental indica que son de alto riesgo para la propagación de incendios.

Así, 62 \% de los expertos estaría dispuesto a participar económicamente en la formación de un fondo para buscar solución a los problemas ambientales, mientras que $92 \%$ estuvo de acuerdo en manifestarse públicamente para lograr su resolución (cuadro 6). 
Cuadro 6. Comparación ENTRE LAS RESPUESTAS DE LA SOCIEDAD Y EL PANEL DE EXPERTOS

\begin{tabular}{lcc}
\hline Formas de solución de los problemas ambientales & $\begin{array}{c}\text { Sociedad } \\
(\%)\end{array}$ & $\begin{array}{c}\text { Expertos } \\
(\%)\end{array}$ \\
\hline Creación de un fondo para la resolución de problemas ambientales & 78.5 & 62 \\
Manifestación publica para la solución de los problema ambientales & 82.1 & 92 \\
\hline
\end{tabular}

\section{Discusión}

La población encuestada y el panel de expertos coinciden en señalar la contaminación del agua subterránea como el principal problema ambiental.

El acuífero de Yucatán tiene la particularidad de ser un acuífero cárstico, con características de alta porosidad, y la presencia de grietas, cenotes y cavernas, que le confieren alta permeabilidad (Bautista et al., 2005); la distancia entre las actividades antrópicas y el acuífero es de uno a 40 metros en más de $60 \%$ de la superficie del estado (Ihl et al., 2007).Además, en esa superficie se concentran las actividades agropecuarias y la población humana, lo que ocasiona que el acuífero sea altamente vulnerable a la contaminación antrópica (Pérez y Pacheco, 2004; Bolio et al., 2006).

El principal riesgo de contaminación de las aguas subterráneas es la materia orgánica proveniente de dos fuentes: a) los asentamientos urbanos (fundamentalmente por la falta de drenaje); $y$ b) las granjas porcinas, avícolas $y$ ranchos ganaderos; por ejemplo, se ha estimado que de los $6095500 \mathrm{~m}^{3}$ anuales de aguas residuales generados en las granjas porcinas, 37 \% no recibe ningún tratamiento, depositándose en cavernas o grutas cercanas a la explotación (fuente puntual) o directamente a los suelos (fuente difusa) (Drucker, Gómez y Magaña, 1999). Ambas situaciones provocan la degradación de los cuerpos de agua subterráneos, que son la principal fuente de abastecimiento de agua potable para las poblaciones humanas.Asimismo, se presentan efectos indirectos de tipo socioeconómico, como enfermedades, pérdidas de lugares de recreo y turísticos, escasez de agua, pérdida de biodiversidad y de recursos acuáticos en general (Drucker, Gómez Magaña, 1999; Drucker et al., 2003; Pacheco, Calderón y Cabrera, 2004; Pacheco, Cabrera, Cabrera y Pérez, 2004; Pérez y Pacheco, 2004). 
A pesar de que la población y los expertos identifican como principal problema ambiental la contaminación del acuífero, las autoridades no han puesto en marcha las medidas de solución. Es bien conocido que hay dos formas de remediar el problema: a) instalar el drenaje doméstico en todas las ciudades; b) hacer valer la legislación en materia de descarga de aguas residuales a las granjas porcícolas y avícolas, los ranchos ganaderos y las empresas del ramo alimentario, entre otros.

El alto costo de la instalación del drenaje ha sido el argumento para justificar su no instrumentación, ya que en las planicies cársticas del estado de Yucatán, a nivel casi superficial, se localiza una capa muy dura de carbonato de calcio denominada laja (figura 3). No hay un estudio económico comparativo entre los costos económicos de instalación del drenaje y los costos (ambientales, sociales y de salud) que paga la población de escasos recursos económicos, que es la que hace uso del acuífero somero. Sin embargo, empresas telefónicas han instalado una red subterránea de fibra óptica, lo cual es un ejemplo de que existe la tecnología para instrumentar el drenaje en las ciudades de Yucatán.

En el caso de las granjas y empresas que descargan aguas residuales al acuífero y que se encuentran fuera de la norma oficial mexicana 00I (Semarnat, 1996a), el argumento para no hacer cumplir la ley es la necesidad de los empleos que estas empresas generan. Ante esta situación, los costos ambientales y de salud seguirán siendo pagados por las clases de menores recursos económicos que, como ya se dijo, usan el agua del acuífero somero para sus necesidades básicas.

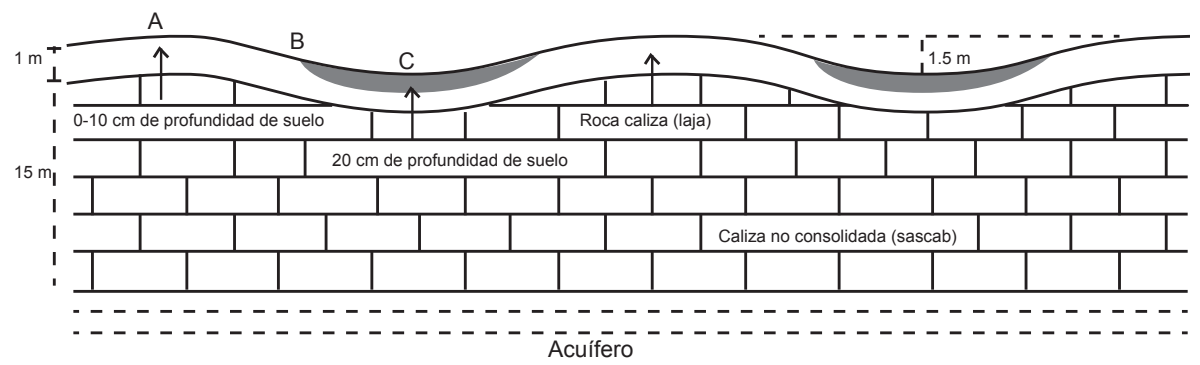

Fuente: (Bautista et al., 2003).

Figura 3. Localización de la laja y del agua subterránea en Mérida, Yucatán. $A=$ LEPTOSOL LITICO, $B=$ LePTOSOL RÉNDZICO Y C = CAMBISOL 


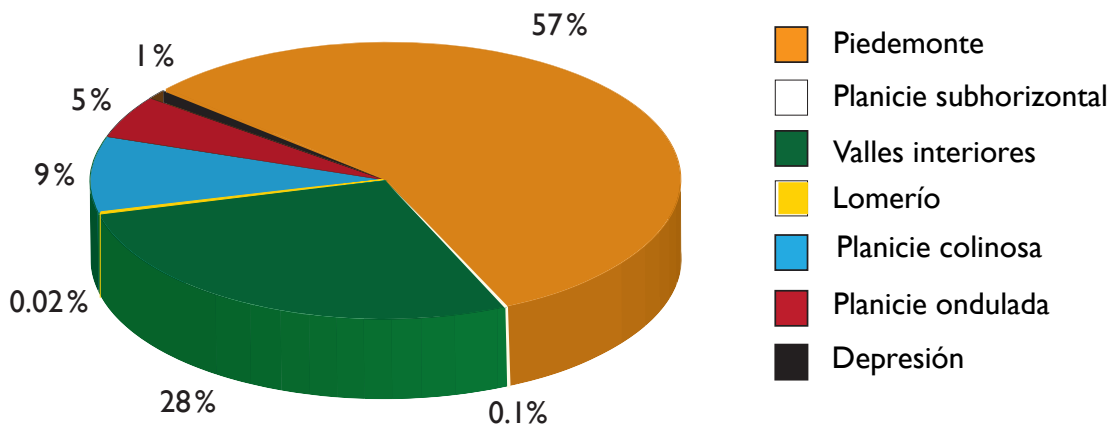

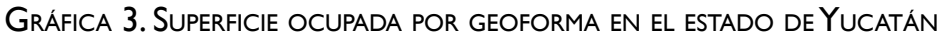

La población encuestada y el panel de expertos coinciden parcialmente en cuanto a la identificación de los principales problemas ambientales. Las diferencias radican en la relevancia de éstos, quizá porque los expertos poseen una visión más amplia de los problemas del estado en comparación con el perfil urbano de los entrevistados. Sin embargo, diversos autores mencionan otro tipo de problemas ambientales como más importantes: la deforestación, el deterioro de los ecosistemas y la degradación del suelo, que a su vez generan fuertes problemas sociales, como la emigración (con su consecuente fragmentación familiar) y la desnutrición infantil, por señalar algunos (Cuanalo y Siniarska, 2006). Esta situación se presenta debido a que la población encuestada e incluso los expertos son de procedencia urbana e identifican plenamente los problemas ambientales de la ciudad de Mérida y de algunos municipios costeros aledaños que ocupan $28 \%$ de la superficie (gráfica 3), ya que existen poblaciones al sur del estado, en los valles interiores (I \%) y lomeríos (9\%), que no tienen como prioridad ambiental la contaminación del acuífero ni la erosión de las playas, sino las inundaciones periódicas por los huracanes (gráfica 3). En las planicies onduladas, más de $70 \%$ de la superficie se encuentra con pastizales que son regados exhaustivamente, lo cual ocasiona la intrusión salina y el deterioro de las selvas baja y mediana (Frausto et al., 2006; lhl et al., 2007).

Tratar los problemas ambientales desde una perspectiva geográfica enriquece el análisis y permite generar una discusión más allá de la descripción del problema, al considerar el medio físico, los usos del recurso, la normatividad 
de uso de los recursos, la percepción social y la búsqueda de posibles soluciones. La distribución geográfica de los problemas clave debe atenderse en ese mismo sentido; lo que es prioritario para algunos no lo es para otros. Se requiere el diálogo multi-, inter- y transdisciplinario, con un enfoque geográfico para una mejor atención a los problemas ambientales.

\section{Conclusiones}

El tamaño de la muestra y la selección de las personas encuestadas son dos factores criticables en este tipo de trabajos. La muestra, considerando el área geográfica bajo análisis, es pequeña, y tal vez no estuvieron suficientemente representados algunos estratos sociales de la población yucateca; sin embargo, los resultados de este trabajo pueden tomarse como un primer acercamiento a la percepción social de los problemas ambientales.

A pesar de las limitaciones indicadas, es posible concluir lo siguiente:

La mayoría de la gente identifica que los principales problemas ambientales son la contaminación del acuífero (6l \%), la erosión de las playas (55\%) y la pérdida de la biodiversidad (35\%). El reconocimiento de la importancia del problema de contaminación del acuífero es determinante, y debería ser tenido en cuenta por los responsables de las políticas públicas correspondientes y la adjudicación de recursos en proyectos específicos.

De los entrevistados, 78.5 \% está dispuesto a aportar recursos económicos para la solución de los problemas ambientales; la disponibilidad de aportación depende de la ocupación, y la aportación mediana global es de 50 pesos. Este hecho es encomiable, e independientemente de que se proceda o no a crear un fondo de recursos basado en la contribución de particulares, indica compromiso social con cuestiones colectivas. Este compromiso debe ser considerado por las autoridades para corresponder de manera honesta y desinteresada en proyectos dirigidos a detener o revertir los problemas señalados.

De la muestra, 82. I\% está dispuesto a manifestarse públicamente para lograr la solución de los problemas ambientales. La decisión de manifestarse es independiente de la edad. La contaminación del acuífero y los residuos son los dos problemas que más motivarían a la gente a manifestarse públicamente. Al igual que en el punto anterior, políticos y funcionarios deben advertir que pueden contar con vastos sectores de la sociedad para implementar 
instrumentos de política que supongan la participación ciudadana, $y$, a la vez, cuidarse de no defraudar la confianza que pueda depositarse en ellos.

De los encuestados, $53 \%$ piensa que las fábricas son las responsables directas de la contaminación; 30 \% responsabiliza al gobierno; y $17 \%$ a la sociedad en general. Una mayor proporción de amas de casa, comerciantes y empleados señalaron a las autoridades como las responsables de resolver los problemas ambientales, mientras que los estudiantes y profesionistas se inclinaron más por la sociedad.

De la población, 59.5 \% preferiría que se atendieran los problemas ambientales en lugar de gastar el dinero en subsidios a la iniciativa privada.

Desde un punto de vista práctico, este diagnóstico ambiental de la sociedad propone que es el momento propicio para tomar decisiones en torno a la búsqueda de medidas de solución de los problemas ambientales, principalmente la contaminación del acuífero y el manejo de los residuos. Desde una perspectiva teórica, el trabajo sugiere la relevancia de la participación social con dos metas claras. La primera, definir prioridades para la política pública ambiental en los niveles de gobierno que corresponda. La segunda, capturar el conocimiento y la percepción social acerca de los problemas permite jerarquizarlos y arribar a sugerencias bien establecidas en órganos como los consejos de cuenca y los organismos de formulación y seguimiento del ordenamiento ecológico y territorial. Por su parte, hay otras zonas en el estado de Yucatán que tienen prioridades distintas en cuanto a sus problemas ambientales.

El contraste entre la información generada mediante las encuestas y el panel de expertos y la publicada por otros investigadores mediante un enfoque geográfico transdisciplinario permitió valorar en su justa dimensión los resultados de este estudio, haciendo notar que, en la percepción de los problemas ambientales, los individuos tienen un sesgo geográfico por el conocimiento de su entorno.

\section{Agradecimientos}

A los participantes en el taller de Impacto Ambiental 2006 por la aplicación de las encuestas y al C.P. César Anaya-Ramírez por la elaboración de la base de datos.A los miembros del Programa Institucional de Medio Ambiente de la Universidad Autónoma de Yucatán por su participación en el panel de expertos. 


\section{FUENTES CONSULTADAS}

Bautista, F. (2005). "Información edafológica para el manejo de recursos naturales", en F. Bautista y G. Palacio (eds.). Caracterización y manejo de los suelos de la Península de Yucatán. Implicaciones agropecuarias, forestales y ambientales. México: Universidad Autónoma de Campeche (UAC)/Universidad Autónoma de Yucatán (UADY)/Instituto Nacional de Ecología (INE), I-8.

— agroindustriales. Caso: vinazas crudas y tratadas. México: Universidad Nacional Autónoma de México (UNAM).

— , et al. (2003). "Microrrelieve y color del suelo como propiedades de diagnóstico en Leptosoles cársticos”. Terra. 2 I: I-I I.

, et al. (2005). "Integración del conocimiento actual sobre los paisajes geomorfológicos de la Península de Yucatán”, en F. Bautista y G. Palacio (eds.). Caracterización y manejo de los suelos de la Península de Yucatán. Implicaciones agropecuarias, forestales y ambientales. México: UAC/UADY/INE, 33-58.

y J. Jiménez (200I). “Consideraciones para el manejo y conservación de recursos naturales en Yucatán”. Revista de la Universidad Autónoma de Yucatán, 16 (2I 7), 40-46.

Bocco, G. (2007). “Algunas reflexiones sobre geografía, ambiente y geografía ambiental". Manuscrito de la ponencia presentada ante la Cátedra de Geografía Humana Eliseo Reclus. México: Instituto Mora.

Bolio, E., et al. (2006). "Caracterización del epikarst en el estado de Yucatán para su uso en el cálculo de la vulnerabilidad del acuífero, mediante el método EPIK". XV Congreso Nacional de Ingeniería Sanitaria y Ciencias Ambientales. Guadalajara, 24-26 de mayo.

Cuanalo, H. y A. Siniarska (2006). "Changes in a Rural Community (Yucatan, Mexico) Associated with Improvements in Production and Productivity". International Journal of Anthropology, 2I, I 3 I- I 40.

Drucker, A., et al. (2003). "La industria porcina en Yucatán: un análisis de la generación de aguas residuales". Problemas del Desarrollo. Revista Latinoamericana de Economía, 34 (I35), I05-I 24.

,V. Gómez y S. Magaña (1999). “¿Contaminante o bien?: Estrategias 
y políticas para el tratamiento de desechos animales". Investigación Económica, 227: III-|4|.

Eden, S. (1998). "Environmental Issues: Knowledge, Uncertainty and the Environment". Progress in Human Geography, 22 (3), 425-432.

Espinoza, G. (2002). Gestión y fundamentos de evaluación de impacto ambiental. Santiago de Chile: Banco Interamericano de Desarrollo/Centro de Estudios para el Desarrollo.

Frausto, O., et al. (2006). “Áreas susceptibles de riesgo en localidades de pobreza extrema en el sur de Yucatán”. Teoría y Praxis, 2, 87-I03.

García, C. y A. Álvarez (2003). “...y sin embargo se vive. De la infravivienda a la vivienda urbana en Mérida, Yucatán, México”. Scripta Nova. Revista Electrónica de Geografia y Ciencias Sociales, VII (I46), I I de agosto [en línea]. Barcelona: Universidad de Barcelona. Disponible en: http:// www.ub.es/geocrit/sn/sn-I46(I43).htm

Ihl, T., et al. (2007). "Identification of Geodisasters in the State of Yucatan, Mexico”. Neues Jahrbuch für Geologie und Paläontologie, 246 (3), 299$3 \mathrm{II}$.

Leff, E. y J. Carabias ( 1993). Cultura y manejo sustentable de los recursos naturales. México: UNAM/Porrúa/Programa de Naciones Unidas para el Medio Ambiente.

Moya, X., et al. (2003). “La agricultura campesina de los mayas en Yucatán”. LEISA. Revista de Agroecología edición especial: 7-I7.

Pacheco, J., A. Cabrera y R. Pérez (2004). "Diagnóstico de la calidad del agua subterránea en los sistemas municipales de abastecimiento en el estado de Yucatán, México”. Ingeniería, 8 (2), I65-I 79.

,L. Calderón yA. Cabrera (2004).“'Delineación de la zona de protección hidrogeológica para el campo de pozos de la planta Mérida I, en la ciudad de Mérida,Yucatán, México”. Ingeniería, 8 (I), 7-I6.

Pérez, R. y J. Pacheco (2004). "Vulnerabilidad del agua subterránea a la contaminación de nitratos en el estado de Yucatán”. Ingeniería 8 (I), 33-42.

Ramírez, R. (1997). Malthus entre nosotros: discursos ambientales y la política demográfica en México 1970-1995. México: Ediciones Taller Abierto/ Universidad Pedagógica Nacional.

Sánchez, L. (2000).“Impactos sobre el medio antrópico”. II Curso Internacional 
de Aspectos Geológicos de Protección Ambiental. Campinas: Organización de las Naciones Unidas para la Educación, la Ciencia y la Cultura.

Santos, J. y G. Molina (2004). “Diagnóstico rural participativo”, en F. Bautista et al. (eds.). Técnicas de muestreo para manejadores de recursos naturales. México: UNAM/INE/UADY, 399-420.

Semarnat (1996a). Norma Oficial Mexicana NOM-00 I-ECOL-I 996 que establece los límites máximos permisibles de contaminantes en las descargas de aguas residuales en aguas y bienes nacionales. Publicada en el Diario Oficial de la Federación el 14 de enero de 1998, México: Secretaría de Medio Ambiente y Recursos Naturales.

(1996b). Manual del proceso de ordenamiento ecológico. México: Secretaría de Medio Ambiente y Recursos Naturales.

Soporte técnico

SPSS (2003). SPSS 12.0 for Windows. Chicago.. 